\title{
LA PRESENCIA DE UNA FAMILIA NEOTROPICAL (MALPIGHIACEAE) EN EL EXTREMO MÁS AUSTRAL DE SUDAMÉRICA DURANTE EL EOCENO
}

\author{
DAMIÁN A. FERNÁNDEZ, CAROLINA PANTI, LUIS PALAZZESI \& VIVIANA D. BARREDA \\ División Paleobotánica, Museo Argentino de Ciencias Naturales "Bernardino Rivadavia", CONICET, \\ Av. Ángel Gallardo 470, C1405DJR, Buenos Aires, Argentina. fernandez.da@hotmail.com, \\ caropanti@macn.gov.ar,lpalazzesi@macn.gov.ar,vbarreda@macn.gov.ar
}

\begin{abstract}
THE PRESENCE OF A NEOTROPICAL FAMILY (MALPIGHIACEAE) IN THE FAR SOUTH OF SOUTH AMERICA DURING THE EOCENE. Presented here is the discovery of pollen grains and a foliar impression assigned to Malpighiaceae from the Río Turbio Formation, SW Santa Cruz Province, indicating the presence of this Neotropical family in the southernmost tip of South America by the Eocene. Perisyncolporites pokornyi Germeraad, Hopping \& Muller, erected to group fossil pollen similar to Malpighiaceae, includes a wide variety of pollen-forms with a complex aperture distribution, more or less concordant with the sides of a hexagon. The extant clades most closely related to the fossil forms of the Río Turbio Formation (Tetrapteroids and Stigmaphylloids) are today distributed in tropical and subtropical regions of South America, reaching up to the $35^{\circ} \mathrm{S}$. The only extant species distributed closer to the studied area, and that, in turn, represents the world's southernmost penetration of the family (Gallardoa fischeri Hicken, reaching the $42^{\circ} \mathrm{S}$ ), is morphologically unrelated to the studied fossils. The foliar impression is similar to those recognized previously in the Río Pichileufú locality, Río Negro Province (Mascagnia sepiumoides Berry, Tetrapteris precrebrifolia Berry), and also in the Río Turbio Formation (Tetrapteris precrebrifolia Berry). The present results support earlier hypotheses that suggest a major penetration of Neotropical lineages into the southernmost latitudes during the Eocene as well as a possible migration to Australia via Antarctica, at least of those perisyncolporate-related forms.
\end{abstract}

Key words: Malpighiaceae, fossil pollen, foliar impression, Eocene, Patagonia.

\section{INTRODUCCIÓN}

Malpighiaceae es una familia de angiospermas representada en el mundo por unas 1260 especies de árboles, arbustos y lianas. El principal centro de diversidad de Malpighiaceae se encuentra en la zona tropical de Sudamérica, región propuesta como el área de origen de la familia (Anderson, 2004). Brasil cuenta con la mayor cantidad de especies de Malpighiaceae en el mundo (Arènes, 1957; Anderson, 2004).

En el registro fósil la familia Malpighiaceae está representada en su mayoría por granos de polen del tipo banisteroide sensu Lowrie (1982). Éstos fueron agrupados en Perisyncolporites pokornyi Germeraad, Hopping \& Muller, 1968, especie documentada fundamentalmente en depósitos paleógenos del norte y centro de Sudamérica (Germeraad et al., 1968; Regali et al., 1974a,b; Lima \& Salard-Cheboldaeff, 1981; Muller, 1981; Lima \& Dino, 1984; Lima \& Amador, 1985; Graham, 1985; Lima et al., 1985,1991,1996; Lima \& Melo, 1994; Garcia et al., 2008; Jaramillo \& Dilcher, 2001; Santos et al., 2010), África (Germeraad et al., 1968) y Australia (Martin, 2002); y en depósitos neógenos (Mioceno) de Argentina (Anzótegui \& Garralla, 1986; Yamamoto, 1995; Leite et al., 1997; Anzótegui \& Aceñolaza, 2008; Mautino, 2010). Su distintiva morfología, y su reconocido rango temporal le confiere a este tipo morfológico un gran valor como indicador estratigráfico (Jaramillo \& Dilcher, 2001). Los registros megascópicos, si bien más escasos, han sido documentados mediante improntas foliares (Berry, 1918, 1938; Hünicken, 1955, 1967; Lott et al., 2011), flores (Taylor \& Crepet, 1987) y frutos (Hably \& Manchester, 2000).

La Formación Río Turbio se encuentra situada en el SO de la Provincia de Santa Cruz (Figura 1), dominio de la Cuenca Austral o Magallánica. Esta cuenca abarca una parte importante de la provincia argentina de Santa Cruz, la chilena de Magallanes, la zona oriental del estrecho homónimo, la isla Grande de la Tierra del Fuego y una porción de la plataforma continental argentina lindante al este de las mismas (Malumián \& Caramés, 1997). Durante el Paleógeno la Cuenca Austral se comportó como una cuenca de antepaís mixta (Biddle et al., 1986), con sedimentación marina, marginal y continental.

En este trabajo se presenta el registro fósil más austral de Perisyncolporites pokornyi y de una impronta foliar asignados a la familia Malpighiaceae en sedimentos de la Formación Río Turbio, asignada al Eoceno medio (Malumián \& Jannou, 2010), momento en cual numerosas familias de angiospermas de actual distribución neotropical alcanzaron su registro más austral. Los granos de polen de Malpighiaceae poseen características únicas que permiten su asignación a nivel de familia de manera confiable. Este hallazgo aporta un nuevo registro, importante desde el punto de vista bioestratigráfico y paleoecológico, y representa una pieza clave para la interpretación de futuras inferencias paleobiogeográficas.

\section{MATERIAL Y MÉTODOS}

Los granos de polen de Malpighiaceae fueron reconocidos a partir del análisis de preparados palinológicos depositados en la colección de Paleopalinología del MACN, bajo la 


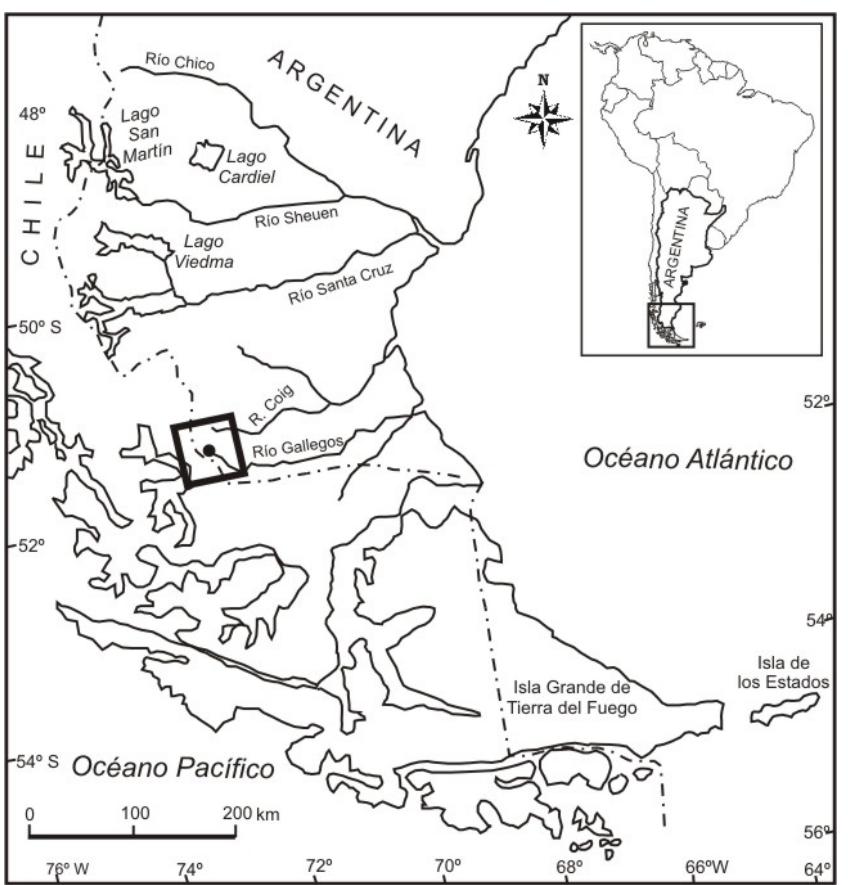

Figura 1. Mapa de ubicación.

Figure 1. Location map.

sigla BAPal ex CIRGEO, y los números de catálogo 98 a 100. Las muestras analizadas provienen del pozo YCF T-134 (51 $\left.35^{\circ} \mathrm{S}, 7^{\circ} 20^{\prime} \mathrm{O}\right)$ de Yacimientos Carboníferos Fiscales, profundidades 612 a $632 \mathrm{mbbp}$ (metros bajo boca de pozo), y se corresponden con la sección inferior de la Formación Río Turbio. Para más detalles sobre la estratigrafía remitirse a Romero (1977). En la descripción morfológica de los granos de polen se sigue la terminología de Punt et al. (2007). Las coordenadas del material ilustrado se corresponden con la reglilla England Finder. Para la determinación de la afinidad botánica se comparó con bibliografía específica y con material depositado en la colección de Actuopalinología (BAPA) del Museo Argentino de Ciencias Naturales "Bernardino Rivadavia" (MACN), así como con material de herbario previamente acetolizado.

El material megaflorístico (hoja) se encuentra preservado a modo de impresión y proviene de la sección inferior de la Formación Río Turbio (Panti, 2010). El material estudiado se encuentra depositado en la colección paleobotánica del Museo Provincial Padre Manuel Jesús Molina (MPMPB) ubicado en la ciudad de Río Gallegos, Provincia de Santa Cruz, Argentina. La descripción sistemática sigue el formato propuesto por Hickey (1977) y Johnson (1989), mientras que la terminología utilizada es la propuesta por Hickey $(1973,1977,1979)$, Dilcher (1974), Hickey \& Wolfe (1975), LAWG (1999) y Ellis et al. (2009). El material fue fotografiado y dibujado para obtener un detalle más preciso del patrón de venación. Para determinar la afinidad taxonómica en base a los caracteres morfológicos se comparó el material con datos bibliográficos y con material actual procedente del herbario del MACN.

\section{PALEONTOLOGÍA SISTEMÁTICA}

\author{
Granos de polen \\ Clase PERICOLPORATAE Iversen \& Troels Smith, 1950
}

Perisyncolporites Germeraad, Hopping \& Muller, 1968

Especie tipo. Perisyncolporites pokornyi Germeraad, Hopping \& Muller, 1968.

Observaciones al género. Perisyncolporites es un morfogénero monotípico definido para agrupar formas fósiles afines a la familia Malpighiaceae. La única especie fósil descripta hasta el momento, P. pokornyi, incluye un amplio rango de formas esféricas a sub-poligonales, con disposición muy compleja de sus aperturas, en general más o menos concordantes con los lados de un hexágono. Dicho patrón puede estar incompleto o totalmente ausente. La gran variabilidad morfológica de la especie ha hecho suponer que P. pokornyi involucre a más de un grupo natural (Germeraad et al., 1968; Martin, 2002). El escaso número de ejemplares fósiles recuperados hasta el momento en la Fm. Río Turbio impide cualquier intento de subdivisión, y el conjunto de estas formas eocenas es asignado en forma amplia a P. pokornyi.

\section{Perisyncolporites pokornyi Germeraad, Hopping \& Muller, 1968}

(Figuras 2A-D)

Material estudiado. Especímenes provenientes de las preparaciones palinológicas BAPal, ex CIRGEO 98, X51-3; BAPal, ex CIRGEO 99, Y37-2; BAPal, ex CIRGEO 100, C43-2; BAPal, ex CIRGEO 100, J40-4.

Descripción. Granos de polen porados con colpoides, con sincolpoides no siempre reconocible, esféricos a subesféricos de contorno subcircular. Poros circulares a subcirculares con márgenes definidos, en número de seis a diez, con al menos tres de ellos asociados a los colpos, aunque no se observa un arreglo definido. Diámetro de los poros variable de 1 a $3 \mu \mathrm{m}$. Colpoides muy poco profundos, sin un patrón determinado, afectando sólo a la sexina, muy delgados, observándose como rajaduras de la exina. Exina de 2 a $3 \mu \mathrm{m}$ de espesor, con estratificación poco definida; columelas no observables. Nexina tres a cuatro veces más espesa que la sexina; sexina muy delicada, superficialmente escabrada.

Dimensiones. Cuatro ejemplares con diámetro: 22 a $30 \mu \mathrm{m}$. Procedencia del material. Preparado BAPal, ex CIRGEO 98, Pozo YCF, T. 134, 612-613 m; Preparado BAPal, ex CIRGEO 99, Pozo YCF, T. 134, 612-613 m; Preparado BAPal, ex CIRGEO 100, Pozo YCF, T. 134, 630-631.5 m, Río Turbio, Santa Cruz.

Comentarios. Si bien estos granos responden al patrón general descripto anteriormente cabe destacar que la escultura del ejemplar ilustrado en la Figura $2 \mathrm{C}$ es más delicada, gradando desde tenuemente escabrada a psilada, aunque esta variación puede deberse a un sesgo tafonómico. Otro carácter a destacar, observado en el ejemplar de la Figura 2D, se refiere a la presencia de pequeños orificios de un diámetro menor a 1 $\mu \mathrm{m}$, dispuestos sin seguir un patrón evidente, contabilizándose 
al menos 15 (flechas de la Figura 2D). Estas perforaciones no parecen representar una estructura funcional del grano y es posible que sean producto algún tipo de corrosión.

Comparaciones. Estos ejemplares presentan similitudes generales con el material tipo definido para el Cenozoico del área del Caribe y Nigeria (Germeraad et al., 1968), aunque se apartan de dicho material por presentar un patrón morfológico más irregular (especialmente en la disposición de las aperturas) y menores dimensiones generales. Algo semejante sucede con los especímenes de Perisyncolporites pokornyi ilustrados para el Eoceno de Brasil (Lima \& Amador, 1985) y de Colombia (Jaramillo \& Dilcher, 2001). Las mayores diferencias con aquellos ejemplares radican en su forma poligonal y en que las aperturas presentan una distribución más simétrica, a la vez que los poros son de diámetros mayores. Por otra parte los ejemplares de Malpighiaceae, asignados a Miocaenipollis, ilustrados (aunque no descriptos) para el Eoceno superior de Brasil (Lima \& Amador, 1985), parecen presentar una esculturación verrucosa.

De los tres tipos polínicos de la especie polimórfica Perisyncolporites pokornyi, reconocidos por Martin (2002) para el Cenozoico de Australia, el tipo morfológico A es el que más se asemeja a nuestro material. Comparten el tipo y disposición de las aperturas, número de poros, grosor de la exina, escultura y diámetro general. Cabe destacar que este tipo morfológico es el que se reconoce en especies actuales en su mayoría nativas de América (Willis, 1966; Martin, 2002). Afinidad botánica. El material analizado presenta similitudes generales con los tipos polínicos reconocidos en la familia Malpighiaceae, especialmente con aquellos del grupo Banisteroide según la filogenia propuesta por Davis \&
Anderson (2010). En líneas generales dentro de este clado comparten semejanzas morfológicas con algunas especies de los clados Tetrapteroide [(Heteropterys glabra Hook. \& Arm. y Callaeum psilophyllum (A. Juss.) D.M. Johnson) y Stigmaphylloide (Stigmaphyllon bonariense (Hook. \& Arm.) C.E. Anderson] (Figura 2E), actualmente presentes en el norte argentino y en la Provincia de Buenos Aires. En términos morfológicos comparten la presencia de colpoides delgados, un número similar de poros y contorno circular a subcircular (sin formas poligonales). Sin embargo, en las especies actuales, las dimensiones generales son mayores y sus aperturas presentan un patrón más regular.

Cabe mencionar que la especie de Malpighiaceae más cercanas geográficamente al área de estudio y que representa la penetración más austral de la familia en el mundo, Gallardoa fischeri Hicken, (clado Cordobioide también incluido dentro del Banisteroide) se aparta del material analizado por presentar una evidente esculturación verrucosa.

$$
\begin{gathered}
\text { Impronta foliar } \\
\text { Morfotipo A } \\
\text { (Figuras 3A-B) }
\end{gathered}
$$

Material examinado. MPMPB 2911.

Localidad fosilífera. Formación Río Turbio, Arroyo Santa Flavia.

Nivel fosilífero. RT4.

Descripción. Hoja simple, de forma elíptica-romboidal y de tamaño micrófilo (longitud de la lámina, 20,4 mm; ancho de la lámina, 12,5 mm). Hoja simétrica; relación largo/ancho
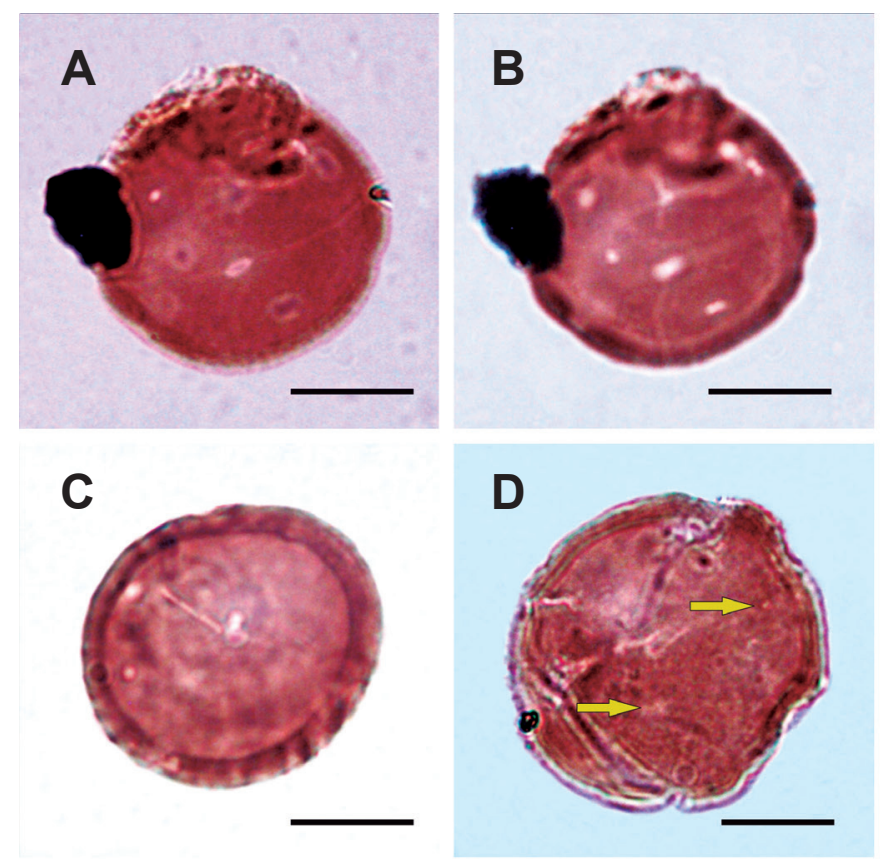

Figura 2. Perisyncolporites pokornyi Germeraad, Hopping \& Muller. A-B, BAPal, ex CIRGEO 100, C43-2, aspecto general (A), foco en las aperturas (B); C, BAPal, ex CIRGEO 100, J40-4, aspecto general; D, BAPal, ex CIRGEO 98, X51-3, apecto general; E, Stigmaphyllon bonariense (Hook. y Arn.) C.E. Anderson, aspecto general. Escalas $=10 \mu \mathrm{m}$.

Figure 2. Perisyncolporites pokornyi Germeraad, Hopping \& Muller. A-B, BAPal, ex CIRGEO 100, C43-2, general aspect (A), focus on the apertures (B); C, BAPal, ex CIRGEO 100, J40-4, general aspect; D, BAPal, ex CIRGEO 98, X51-3, general aspect; E, Stigmaphyllon bonariense (Hook. y Arn.) C.E. Anderson, general aspect. Scale bars $=10 \mu \mathrm{m}$. 

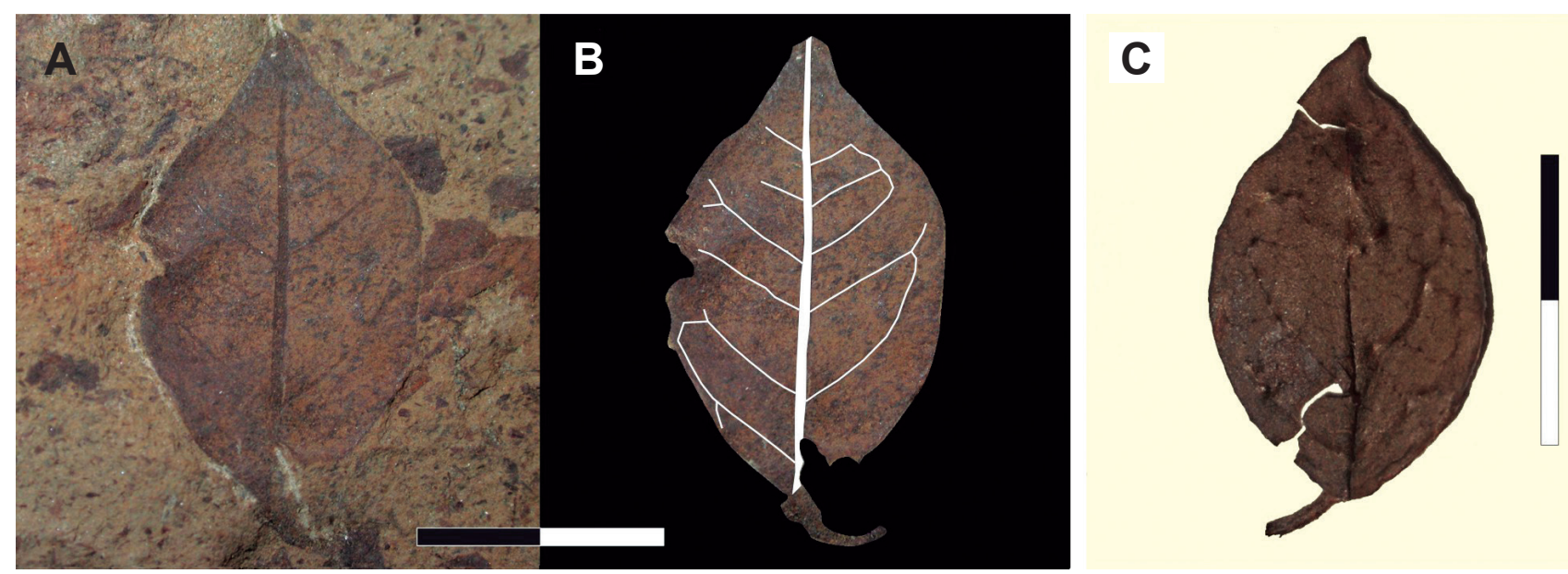

Figura 3. A-B, Morfotipo A (MPMPB 2911); C, Tetrapteris schiedeana (BA 18437). Escalas =10 mm.

Figure 3. A-B, Morphotype A (MPMPB 2911); C, Tetrapteris schiedeana (BA 18437). Scale bars =10 $\mathrm{mm}$.

1,64:1. Ápice acuminado, ángulo apical agudo $\left(78^{\circ}\right)$; base de cuneada a redondeada, ángulo basal agudo $\left(53^{\circ}\right)$. Margen entero. Peciolo marginal y fuertemente curvado. Venación primaria pinnada, vena primaria curvada hacia el ápice; venas secundarias broquidódromas, dispuestas en al menos cuatro pares, el espaciamiento entre las secundarias disminuye hacia el ápice, venas secundarias curvadas distalmente, divergen de la vena media en ángulo agudo $\left(54^{\circ}\right)$; par basal más agudo que los superiores; no se observa la venación de orden mayor. Comparaciones. Las formas fósiles que más se asemejan desde el punto de vista morfológico al morfotipo descripto en este trabajo son las reconocidas para la localidad fosilífera de Río Pichileufú (Formación Huitrera), provincia de Río Negro (Berry, 1938). Estas son Mascagnia sepiumoides (p. 85, lám. 23, figs. 5,6) y Tetrapteris precrebrifolia (p. 84, lám. 22, figs. 3-5). La primera (M. sepiumoides) comparte la forma de la lámina, de la base y del ápice, la presencia de un peciolo fuertemente curvado, de una vena primaria pinnada curvada levemente hacia el ápice y, de cuatro a cinco pares de venas secundarias broquidódromas dispuestas en ángulo agudo con respecto a la vena media. La segunda (T. precrebrifolia), posteriormente reconocida para la Formación Río Turbio (Hünicken, 1955), comparte con el material estudiado la forma de la lámina, el patrón de venación y la presencia de un peciolo marginal fuertemente curvado; sólo se apartaría por el curso de la vena primaria, el cual es más curvado en el material original, y que determina un mayor desarrollo de la lámina sobre su lado convexo.

Afinidad botánica. Si bien para establecer una relación más estrecha es necesario realizar estudios que muestren la presencia de caracteres diagnósticos, el patrón de venación junto con la forma romboidal de la lámina del material analizado son rasgos que pueden ser observados en varias especies de Malpighiaceae actuales. Entre éstas, se pueden observar similitudes con varios géneros de los clados Banisteroide y Tetrapteroide. En especial, son notorias las similitudes en cuanto al patrón de venación, forma de la lámina, inserción y forma de pecíolo que presenta con Tetrapteris schiedeana Cham. \& Schltdl. (Figura 3C), especie ampliamente distribuida en los bosques tropicales, subtropicales y sabanas (Anderson, 1990).

\section{CONSIDERACIONES FINALES}

El presente hallazgo confirma la presencia de la familia Malpighiaceae en el extremo austral de Sudamérica durante el Eoceno. Los clados más afines a las formas fósiles de la Formación Río Turbio están actualmente distribuidos en zonas tropicales y subtropicales de Sudamérica, llegando sólo a latitudes de $35^{\circ} \mathrm{S}$ (Figura 4). La única especie actual con mayor tolerancia a condiciones extremas, Gallardoa fischeri, que alcanza una latitud de $42^{\circ} \mathrm{S}$, se aparta morfológicamente del material estudiado tal cual se expresara anteriormente.

Perisyncolporites pokornyi es un taxón polimórfico que presenta una afinidad botánica múltiple dentro de la familia Malpighiaceae. De esta manera, la especie y sus registros requieren una revisión para lograr una uniformidad de criterios y una afinidad botánica más rigurosa.

El megafósil descripto forma parte de una asociación paleoflorística dominada por morfotipos foliares de margen entero (Panti, 2010). El análisis paleoclimático de la sección inferior de la Formación Río Turbio, de donde proviene el material analizado, indica condiciones de paleotemperaturas cercanas a las que actualmente se desarrollan en la Provincia de Misiones $\left(\mathrm{TMA}=18 \pm 1,5^{\circ} \mathrm{C}\right)($ Panti, 2010). Estos resultados estarían sustentando previas hipótesis sobre una importante penetración de linajes neotropicales en altas latitudes durante el Eoceno.

Por otro lado, la presencia de Malpighiaceae en el extremo más austral de Patagonia sustentaría la hipótesis propuesta por Martin (2002) sobre una posible ruta migratoria de la familia desde Sudamérica hacia Australia a través de Antártida, al menos para las formas perisincolporadas, que son típicamente Americanas. Las condiciones climáticas imperantes en el sur de Sudamérica habrían favorecido la ruta migratoria planteada.

A comienzos del Neógeno, la aridización y el enfriamiento climático trajeron aparejados la extinción local de géneros tropicales en Patagonia (Barreda \& Palazzesi, 2007), haciendo 


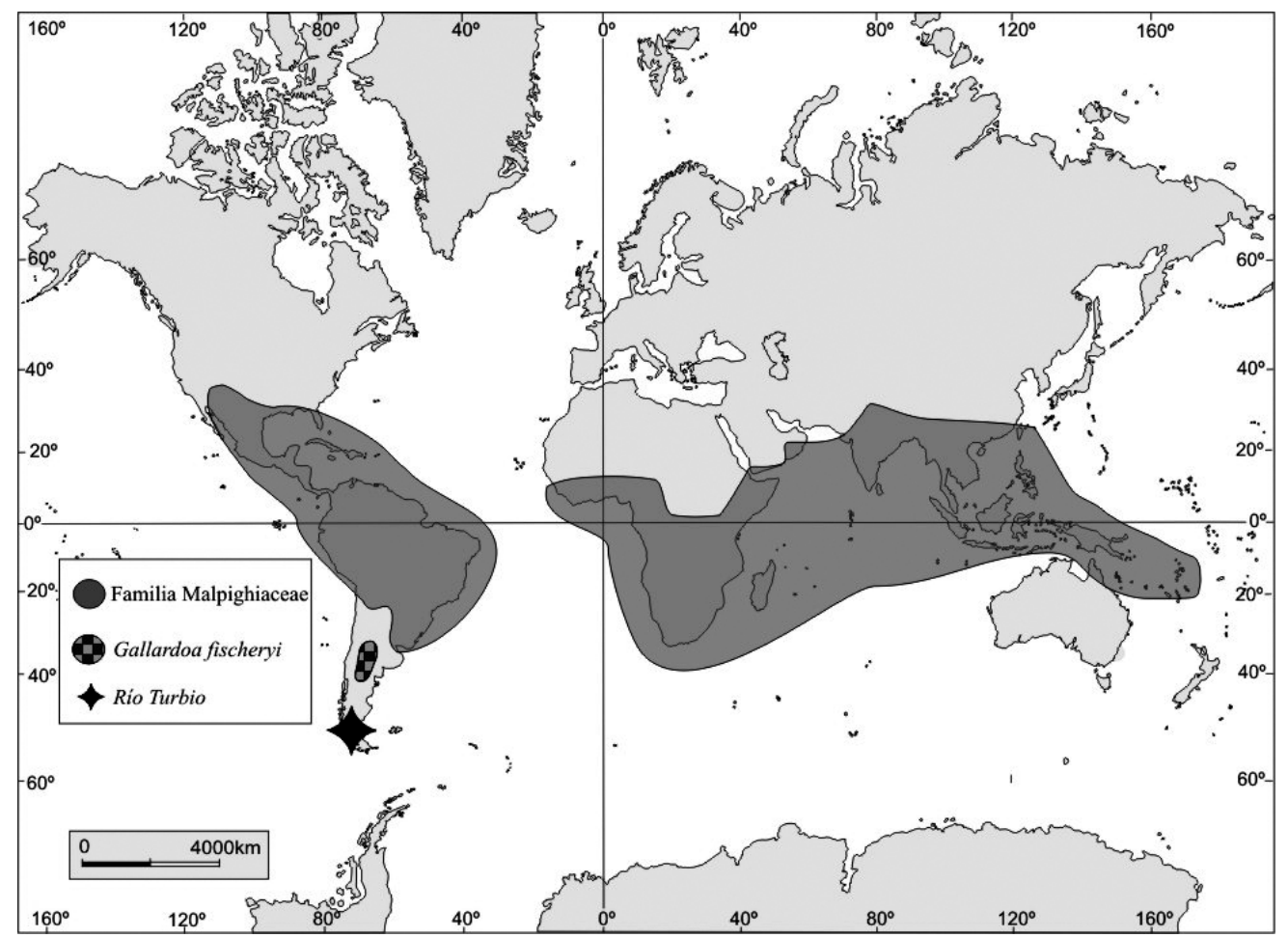

Figura 4. Distribución actual de la familia Malpighiaceae (modificado de Arènes, 1957).

Figure 4. Extant distribution of Malpighiaceae (modified from Arènes, 1957).

que algunos de éstos se desplacen hacia latitudes más bajas. En el caso de las Malpighiaceae, el registro polínico durante el Neógeno se localiza hacia el norte y noreste de Argentina (Anzótegui \& Aceñolaza, 2008; Mautino, 2010) lo que podría estar indicando una posible ruta migratoria desde el Eoceno al Mioceno condicionada por el deterioro climático.

\section{AGRADECIMIENTOS}

Los autores agradecen a los herbarios del Museo Argentino de Ciencias Naturales y del Museo de La Plata por el préstamo de ejemplares herborizados de Malpighiaceae. A M.C. Tellería por la ayuda brindada en la preparación del polen actual y a C. Jaramillo y L. Mautino que contribuyeron, en calidad de árbitros, a mejorar el manuscrito. Este trabajo es una contribución al PIP CONICET 0342.

\section{REFERENCIAS}

Anderson, W.R. 1990. The origin of the Malpighiaceae - the evidence from morphology. Memoirs of the New York Botanical Garden, 64:210-224.

Anderson, W.R. 2004. Malpighiaceae (Malpighia family). In: N. Smith; S.A. Mori; A. Henderson; D.W. Stevenson \& S.V. Heald (eds.) Flowering plants of the neotropics, Princeton University Press in association with The New York Botanical Garden, p. 229-232. doi:10.1600/0363644053661878

Anzótegui, L.M. \& Aceñolaza P. 2008. Macrofloristic assemblage of the Paraná Formation (Middle-Upper Miocene) in Entre Ríos (Argentina). Neues Jahrbuch für Geologie und Paläontologie, Abhandlungen, 248:159-170. doi:10.1127/0077-7749/2008/0248-0159

Anzótegui, L.M., \& Garralla, S. 1986. Estudio palinológico de la Formación Paraná (Mioceno superior) (Pozo Josefina, Provincia de Santa Fe, Argentina). Parte 1. Descripciones sistemáticas. Facena, 6:101-176.

Arènes, J. 1957. Répartition géographique des Malpighiacées vivantes et fossiles (atlas de Biogéographie). Compte rendue sommaire des Séances de la Société de Biogéographie, 290:81-108.

Barreda, V.D. \& Palazzesi, L. 2007. Patagonian vegetation turnovers during the Paleogene-early Neogene: origin of arid adapted floras. The Botanical Review, 73:31-50. doi:10.1663/00068101(2007)73[31:PVTDTP]2.0.CO;2

Berry, E.W. 1918. The fossil higher plants from the Canal Zone. United States National Museum Bulletin, 103:15-44.

Berry, E.W. 1938. Tertiary flora from the Río Pichileufú, Argentina. Geological Society of America Special Papers, 12:1-149.

Biddle, K.; Uliana, M.; Mitchum, R.; Fitzgerald, M. \& Wright, R. 1986. The stratigraphic and structural evolution of the central and eastern Magallanes Basin, southern South America. International Association of Sedimentology, Special Publication, 8:41-61. doi:10.1002/9781444303810.ch2

Davis, C.C. \& Anderson, W.R. 2010. A complete generic phylogeny of Malpighiaceae inferred from nucleotide sequence data and morphology. American Journal of Botany, 97:2031-2048. doi:10.3732/ajb.1000146

Dilcher, D.L. 1974. Approaches to the identification of angiosperm leaf remains. The Botanical Review, 40:1-157.

Ellis, B.; Daly, D.C.; Hickey, L.J.; Johnson, K.K.; Mitchell, J.D.; Wilf, P. \& Wing, S.L. 2009. Manual of Leaf Architecture. Ithaca, Cornell University Press, 216 p. doi:10.1600/036364409790139682

Garcia, M.J.; Bistrichi, C.A.; Saad, A.R.; Campanha, V.A. \& Oliveira, E.P. 2008. Stratigraphy and palaeoenvironments of the Tanque Basin, southeastern Brazil. Revista Brasileira de Paleontologia, 11:147-168. doi:10.1072/rbp.2008.3.02

Germeraad, J.H.; Hopping, C.A. \& Muller, J. 1968. Palynology of tertiary sediments from tropical areas. Review of Palaeobotany and Palynology, 6:189-348. doi:10.1016/0034-6667(68)90051-1 
Graham, A. 1985. Studies of neotropical paleobotany. IV. The Eocene communities of Panama. Annals of the Missouri Botanical Garden, 72:504-534. doi:10.2307/2399101

Hably, L. \& Manchester, S.R. 2000. Fruits of Tetrapterys (Malpighiaceae) from the Oligocene of Hungary and Slovenia. Review of Palaeobotany and Palynology, 111:93-101. doi:10.1016/S0034-6667(00)00019-1

Hickey, L.J. 1973. Classification of the architecture of dicotyledoneus leaves. American Journal of Botany, 60:17-33.

Hickey, L.J. 1977. Stratigraphy and paleobotany of the Golden Valley Formation (Early Tertiary) of western North Dakota. The Geological Society of America Memoir, 150:1-183.

Hickey, L.J. 1979. A revised Classification of the Architecture of Dicotyledonous leaves (25-39). In: C.R. Metcalfe \& L. Chalk (eds.) Anatomy of Dicotyledonous, Clarodendron Press, v. 1, p. 25-39.

Hickey, L.J. \& Wolfe, J.A. 1975. The bases of Angiosperm phylogeny. Vegetative morphology. Annals of the Missouri Botanical Garden, 62:538-589. doi:10.2307/2395267

Hünicken, M. 1955. Depósitos Neocretácicos y Terciarios del Extremo SSW de Santa Cruz (Cuenca carbonífera de Río Turbio). Revista del Instituto Nacional de Investigaciones de las Ciencias Naturales (Museo Argentino de Ciencias Naturales "Bernardino Rivadavia”). Ciencias Geológicas, 4:1-164.

Hünicken, M. 1967. Flora Terciaria de los estratos de Río Turbio, Santa Cruz (niveles plantíferos del Arroyo Santa Flavia). Revista Facultad de Ciencias Exactas, Físicas y Naturales, Universidad Nacional de Córdoba, 27:139-260.

Jaramillo, C.A. \& Dilcher, D.L. 2001. Middle Paleogene palynology of central Colombia, South America: a study of pollen and spores from tropical latitudes. Palaeontographica Abteilung B, 258:87-213.

Johnson, K.R. 1989. High-resolution megafloral biostratigraphy spanning the Cretaceous-Tertiary boundary in the Northern Great Plains. Yale University, Ph.D. thesis, 556 p.

LAWG (Leaf Architecture Working Group), 1999. Manual of leaf architecture: morphological description and categorization of dicotyledonous and net-veined monocotyledonous angiosperms. Washington DC, Smithsonian Institution Press, 65 p.

Leite, F.P.R.; Bernardes-de-Oliveira; Oliveira, P. E.; SilvestreCapelato, M.E.; Arai, M. \& Truckendrodt, W. 1997. Palinofloras miocenas da Formação Pirabas e Grupo Barreiras, na região Bragantina, Estado do Pará, Brasil. Revista Universidade Guarulhos, Geociências, 2:128-140.

Lima, M.R. \& Amador, E.S. 1985. Análise palinológica de sedimentos da Formação Resende, Terciário do Estado do Rio de Janeiro, Brasil. In: Campos, D.A.; Ferreira, C.S.; Brito, I.M. \& Viana, C.F. (eds) Coletânea de Trabalhos Paleontológicos, Brasília, Departamento Nacional da Produção Mineral, p. 371378 (Série Geologia 27, Seção Paleontologia e Estratigrafia 2).

Lima, M.R.; Cabral Jr., M. \& Stefani, F.L.1996. Palinologia de sedimentos da Formação Macacu-Rifte da Guanabara, Terciário do Estado do Rio de Janeiro. Anais da Academia Brasileira de Ciências, 68:531-543.

Lima, M.R. \& Dino, R. 1984. Palinologia de amostras da Bacia de Bonfim, Terciário do Estado de São Paulo, Brasil. Boletim do Instituto de Geociências, 15:1-11.

Lima, M.R.; Melo, M.S. \& Coimbra, A.M. 1991. Palinologia de sedimentos da Bacia de São Paulo, Terciário do Estado de São Paulo, Brasil. Revista do Instituto Geológico, 12:7-20.

Lima, M.R. \& Melo, M.S. 1994. Palinologia de depósitos rudáceos da região de Itatiaia, Bacia de Resende, RJ. Geonomos, 2:12-21.

Lima, M.R. \& Salard-Cheboldaeff, M. 1981. Palynologie des bassins de Gandarela et Fonseca (Eocene de 1'etat de Minas Gerais, Bresil). Boletim do Instituto de Geociências, 12:33-54.
Lima, M.R.; Salard-Cheboldaeff, M. \& Suguio, K. 1985. Étude palynologique de la Formation Tremembé, Tertiairie du Bassin de Taubaté, (Etat de São Paulo, Brésil), d'après les echantillons du sondage no. 42 du CNP. In: Campos, D.A.; Ferreira, C.S.; Brito, I.M. \& Viana, C.F. (eds) Coletânea de Trabalhos Paleontológicos, Brasília, Departamento Nacional da Produção Mineral, p. 379-393. (Série Geologia 27, Seção Paleontologia e Estratigrafia 2).

Lott, T.A.; Dilcher, D.L.; Horn, S.P.; Vargas, O. \& Sanford, R.L. 2011. Pleistocene flora of Rio Puerto Viejo, Costa Rica. Palaeontologia Electronica, 14:1-15.

Lowrie, S.R. 1982. The palynology of the Malpighiaceae and its contribution to family systematics. University of Michigan, Ph.D. thesis, 354 p.

Malumián, N. \& Caramés,A. 1997. Upper Campanian-Paleogene from the Río Turbio coal measures in southern Argentina: micropaleontology and the Paleocene/Eocene boundary. Journal of South America Earth Sciences, 10:189-201. doi:10.1016/S0895-9811(97)00015-1

Malumián, N. \& Jannou, G. 2010. Los Andes Fueguinos: el registro micropaleontológico de los mayores acontecimientos paleoceanográficos australes del Campaniano al Mioceno. Andean Geology, 37:345-374.

Martin, H.A. 2002. History of the family Malpighiaceae in Australia and its biogeographic implications: evidence from pollen. Australian Journal of Botany, 50:171-182. doi:10.1071/BT01039

Mautino, L.R. 2010. Palinofloras de las Formaciones San José y Chiquimil (MiocenoMedio y Superior), Noroeste de Argentina. Universidad Nacional del Nordeste, Tesis Doctoral, 444 p.

Muller, J. 1981. Fossil pollen records of extant angiosperms. The Botanical Review, 47:1-142. doi:10.1007/BF02860537

Panti, C. 2010. Diversidad florística durante el Paleógeno en Patagonia Austral. Facultad de Ciencias Exactas y Naturales, Universidad de Buenos Aires, Tesis Doctoral, 210 p.

Punt, W.; Hoen, P.P.; Blackmore, S.; Nilsson, S. \& Le Thomas, A. 2007. Glossary of pollen and spore terminology. Review of Palaeobotany and Palynology, 143:1-81. doi:10.1016/j.revpalbo.2006.06.008

Regali, M.S.P.; Uesugui, N. \& Santos, A.S. 1974a. Palinologia dos sedimentos meso-cenozoicos do Brasil (I). Boletim Técnico da Petrobras, 17:177-191.

Regali, M.S.P.; Uesugui, N. \& Santos, A.S. 1974b. Palinologia dos sedimentos meso-cenozoicos do Brasil (II). Boletim Técnico da Petrobras, 17:263-301.

Romero, E.J. 1977. Polen de gimnospermas y fagáceas de la Formación Río Turbio (Eoceno), Santa Cruz, Argentina. Buenos Aires, Fundación para la Educación, la Ciencia y la Cultura, 221 p.

Santos, D.; Garcia, M.J.; Saad, A.R. \& Bistrichi, C.A. 2010. Palinoestratigrafia da formação Itaquaquecetuba, Bacia de São Paulo, Brasil. Revista Brasileira de Paleontologia, 13:205-220. doi:10.4072/rbp.2010.3.05

Taylor, D.W. \& Crepet, W.L. 1987. Fossil floral evidence of Malpighiaceae and an early plant-pollinator relationship. American Journal of Botany, 74:274-286.

Willis, J.C. 1966. A dictionary of flowering plants and ferns. $7^{\mathrm{a}}$ ed. (Revised by H.K. Airy Shaw), Cambridge, The University Press, 1214 p.

Yamamoto, I.T. 1995. Palinologia das bacias tafrogênicas do sudeste (bacias de Taubaté, São Paulo e Resende): análise bioestratigráfica integrada e interpretação paleoambiental. Instituto de Geociências e Ciências Exatas, Universidade Estadual Paulista, Dissertação de Mestrado, 217 p.

Received in March, 2012; accepted in September, 2012. 